\title{
Familial pancreatic adenocarcinoma: association with diabetes and early molecular diagnosis
}

James P Evans, Wylie Burke, Ru Chen, Robin L Bennett, Rodney A Schmidt, E Patchen Dellinger, Michael Kimmey, David Crispin, Teresa A Brentnall, David R Byrd

Division of Medical

Genetics,

Department of

Medicine,

Campus Box 7100,

University of North

Carolina,

Chapel Hill,

NC 27599, USA

$\mathrm{J} P$ Evans

Division of General Internal Medicine,

Department of

Medicine,

University of

Washington,

Seattle,

Washington 98195,

USA

W Burke

Department of

Surgery,

University of

Washington,

Seattle,

Washington 98195 ,

USA

R Chen

E P Dellinger

D R Byrd

Department of

Medical Genetics,

Department of

Medicine

University of

Washington,

Seattle,

Washington 98195 ,

USA

R L Bennett

Department of

Pathology,

University of

Washington,

Seattle,

Washington 98195 ,

USA

R A Schmidt

D Crispin

Department of

Gastroenterology,

University of

Washington,

Seattle,
Washington 98195 ,

USA

M Kimmey

T A Brentnall

Correspondence to: Dr Brentnall.

Received 23 August 1994 Revised version 22 December 1994

\begin{abstract}
We report a large pedigree in which pancreatic cancer is inherited in an autosomal dominant fashion. Diabetes and exocrine insufficiency was observed in all family members who eventually developed pancreatic cancer. The presence of diabetes, often years before the diagnosis of cancer, allowed identification of those people who had inherited the predisposing allele and who were thus at high risk for the development of malignancy. This family shows that genetic factors can have a striking effect on the development of pancreatic malignancy and diabetes mellitus. Moreover, preclinical diagnosis of pancreatic cancer in family members provided a unique opportunity to study early molecular changes that accompany the development of human pancreatic cancer. Finally, the molecular approach applied here to the early diagnosis of pancreatic cancer may prove valuable in this family for identification of subjects at risk.
\end{abstract}

( $\mathcal{F}$ Med Genet 1995;32:330-335)

The study of families in which a predisposition to cancer is inherited in a mendelian fashion has lent considerable insight into our understanding of the molecular basis of cancer. ${ }^{1}$ Pancreatic cancer is the fourth leading cause of adult cancer mortality in the United States ${ }^{2}$ and is difficult to diagnose by traditional imaging techniques in its early stages. Moreover, because of the difficulty in diagnosing pancreatic cancer until it is at an advanced stage, it has not been previously possible to observe early molecular changes that are associated with development of this malignancy.

We report here an extensive kindred in which susceptibility to pancreatic cancer is inherited in an autosomal dominant fashion. The presence of insulin dependent diabetes mellitus (IDDM) and exocrine insufficiency in family members destined to develop pancreatic cancer served as a marker for those subjects likely to have inherited the mutant allele, and led to early identification of those at high risk within this pedigree. Based on their perceived high risk of developing pancreatic cancer, three family members with IDDM and exocrine insufficiency elected to undergo prophylactic pancreatectomy. Histologically evident pancreatic adenocarcinoma was observed in two of these people, and molecular analysis showed the presence of mutations in the K-ras protooncogene in pancreatic tissue from all three family members. Molecular analysis of pancreatic secretions obtained by endoscopic retrograde cholangiopancreatography (ERCP) from one of these people allowed preoperative identification of a mutant K-ras proto-oncogene.

The preclinical diagnosis of pancreatic cancer in these subjects provided a unique opportunity to study early molecular changes that accompany the development of human pancreatic cancer. While the clinical and molecular approach applied here to the early diagnosis of pancreatic cancer may prove valuable in this family for identification of people at risk, the death of at least one of these people was probably hastened by medical intervention and serves as a cautionary reminder of the limitations of therapeutic options in pancreatic cancer.

\section{Materials and methods}

DNA EXTRACTION

DNA from pancreatic tissue was obtained using standard extraction methods on $50 \mu$ sections from paraffin blocks. ${ }^{3}$ For extraction of DNA from pancreatic secretions, $50 \mu \mathrm{l}$ of fluid was added to $350 \mu \mathrm{l}$ of DNA extraction buffer and incubated at $60^{\circ} \mathrm{C}$ for 24 to 48 hours, followed by phenol and chloroform extraction.

\section{POLYMERASE CHAIN REACTION (PCR)}

AMPLIFICATION

Exon 1 of K-ras was amplified from the samples using an upstream primer of sequence: $5^{\prime}$ GTACTGGTGGAGTATT $3^{\prime}$ and a downstream primer of sequence: $5^{\prime}$ CTCATGAAAATGGTCAG $3^{\prime}$. Oligonucleotide primers were synthesised in our laboratory on an ABI PCR Mate (Applied Biosystems) using the phosphoramidite method. Template DNA (100 ng) was included in a $50 \mu \mathrm{l}$ solution con- 


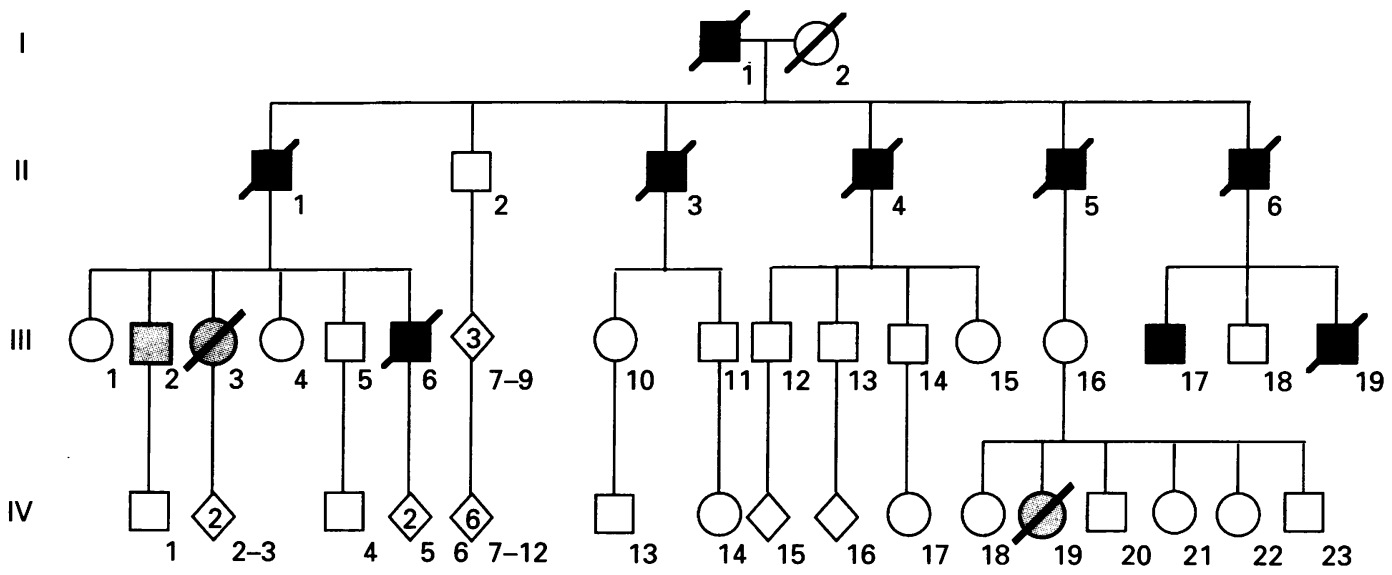

$=$ Pancreatic cancer

$=$ Insulin dependent diabetes mellitus

Figure 1 Pedigree of the proband (III.6)

taining $1 \times$ PCR buffer (Promega, Madison, WI), $2 \mathrm{mmol} / 1 \mathrm{MgCl}_{2}, 100 \mu \mathrm{mol} / 1 \mathrm{dNTP}$ (Pharmacia), $50 \mathrm{ng}$ of each oligomer, and 0.2 units of Taq DNA Polymerase (Promega, Madison, WI). PCR was performed using a cycle profile of $98^{\circ} \mathrm{C}$ (20 seconds), $55^{\circ} \mathrm{C}$ (one minute), and $72^{\circ} \mathrm{C}$ ( 40 seconds) for 35 cycles. Amplification yielded a 290 base pair product containing codons 12 and 13 of K-ras; products were visualised after electrophoresis on a $4 \%$ agarose gel and staining with ethidium bromide.

\section{DNA SEQUENCING}

After amplification, PCR products were purified using a combination of elution and PCR Magic Preps (Promega). Direct sequencing of PCR products was performed using Sequenase (USB) and $\alpha^{32} \mathrm{P}$ labelled sequencing primers with the following sequences: $5^{\prime}$ AAAATGACTTGAATATAAACTTGTG 3' (upstream primer) and $5^{\prime}$ TATTAAAACAAGATTTACC $3^{\prime}$ (downstream primer). Sequencing reactions were electrophoresed on a $6 \%$ polyacrylamide gel, dried, and exposed to film for 6 to 30 hours.

\section{Pedigree}

The pedigree (fig 1) consisted of 50 subjects spanning four generations. Pancreatic cancer had occurred in nine people. Two of the cases in generation II and all of those in generation III had pathological confirmation of their diagnosis; in the others the diagnosis was based on clinical and radiographic findings. Ages at diagnosis ranged from 31 (III-6) to 57 (II·3). All subjects who eventually developed pancreatic cancer had IDDM at the time of the diagnosis of malignancy. Five of the subjects had IDDM for over 10 years before their diagnosis of malignancy (II 1 , II $\cdot 3$, II $\cdot 4$, III 6 , and III 19 ). In four people, IDDM was diagnosed at or about the time of discovery of malignancy $(I \cdot 1, I I \cdot 5$,
II 6 , and III.17). As depicted in fig 1, three subjects in the family were diagnosed with IDDM but have, as yet, not developed pancreatic cancer; subject III 2 recently developed IDDM at the age of 32 , one subject died in a diabetic ketotic coma at age 27 (III. 3 ), and the other family member is described in detail below (IV·19, case 2 ).

At least six of the nine people who developed pancreatic cancer in this family also had a history clinically compatible with pancreatic exocrine insufficiency before diagnosis of cancer. Criteria for a clinical diagnosis of exocrine insufficiency included weight loss, fatty, foul smelling stools, quantitation of faecal fat, and relief with exogenous pancreatic enzyme supplementation. The history of exocrine insufficiency, like that of the IDDM, appears usually to predate the diagnosis of malignancy by as long as several years. None of the people in this pedigree related a medical history compatible with a diagnosis of chronic or acute pancreatitis. There is no history of other types of cancer in this family.

\section{Cases}

CASE 1 (III·6)

The proband, a 31 year old white male, sought genetic counselling regarding his risk of developing pancreatic cancer. He had been insulin dependent secondary to diabetes mellitus since the age of 18, and had recently developed pancreatic exocrine insufficiency. He was otherwise in good health with no complaints.

Inspection of the proband's family history led us to suspect that a gene conferring a high risk of pancreatic cancer was inherited in this family in an autosomal dominant manner, and that the proband's a priori risk of developing pancreatic cancer was as high as $50 \%$. Inspection of the pedigree also suggested that IDDM was a marker for subsequent development of pancreatic cancer in members of 


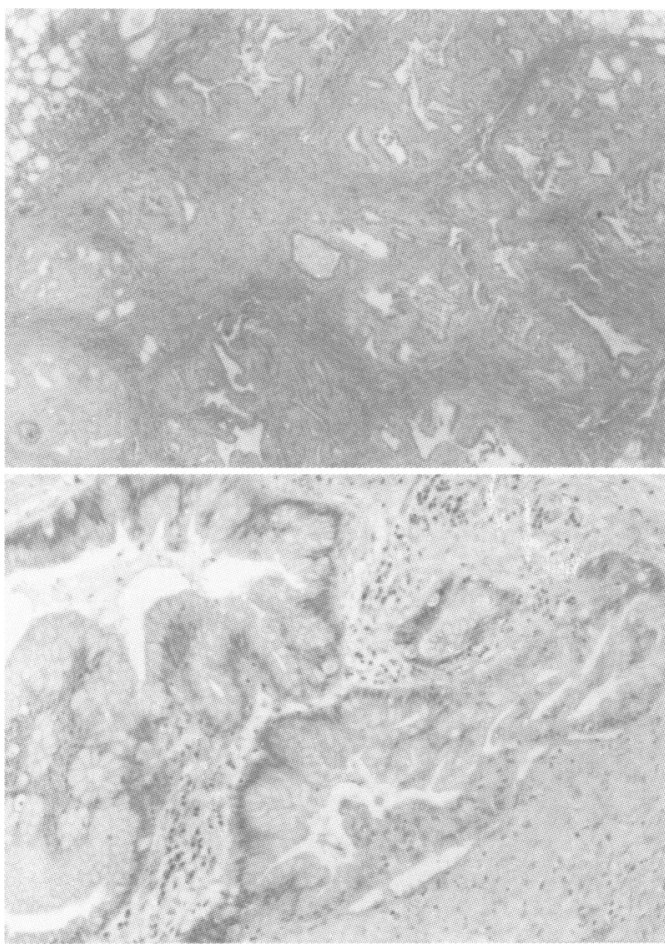

Figure 2 Case 1 (III.6). (Above) Survey photomicrograph showing the pattern of diffuse pancreatic atrophy and fibrosis characteristic of this family. Each lobule appears to undergo acinar atrophy, intralobular fibrosis, and microcystic dilatation of the lobular duct. Cellular inflammation is variable but usually mild (HE्E). (Below) Mucinous epithelial neoplasia. There is epithelial hyperplasia with cytologic atypism in the dilated duct-like structures. The desmoplastic stromal reaction (lower left) and small distorted epithelial structures are suggestive of microscopic invasion (HE⿱E$E)$.

this family, and thus the proband's actual risk for pancreatic cancer was assessed at greater than $50 \%$.

Computerised tomography (CT) of the abdomen with and without contrast was normal Following consultation with the endocrinology, gastroenterology, and surgical services, the patient elected to undergo a prophylactic total pancreatectomy. The operation was uneventful except for the rupture of a pancreatic cyst during surgery.

Pathological examination of the pancreas showed extensive hyperplasia and dysplasia of mucinous type epithelium superimposed on a background of widespread acinar atrophy, dilatation of lobular ducts, inflammation, and fibrosis (fig 2). The neoplastic epithelium tended to line large cystic spaces, including the cyst which ruptured intraoperatively. There was a $1 \mathrm{~cm}$ focus of invasive well differentiated mucinous adenocarcinoma in the head of the pancreas that did not extend to the surgical margins and there was no evidence of nodal metastasis.

The patient did well postoperatively and returned to work within one month of surgery. However, 18 months later he developed ascites and an exploratory laparotomy showed diffuse peritoneal implants of mucinous adenocarcinoma pathologically consistent with pancreatic origin. The patient died eight weeks later.
CASE $2($ IV 19$)$

This patient sought genetic counselling following the discovery of pancreatic cancer in the proband, her first cousin once removed. This 20 year old woman had a 10 year history of IDDM. Several months before her evaluation, she began to lose weight, experience increasing fatigue, and manifest signs of pancreatic exocrine insufficiency. Physical examination showed a thin white woman with retinal changes consistent with diabetic retinopathy. Laboratory studies showed increased faecal fat. A CT scan of the abdomen showed a small, diffusely dense, heavily calcified pancreas with no evidence of a mass.

Pedigree analysis showed that the maternal grandfather of case 2 had died of pancreatic cancer at the age of 31 , leading to an a priori risk of $25 \%$ that she had inherited the predisposing allele. Her mother (III-16) was unavailable for evaluation, but was reported to be free of IDDM. In spite of her mother's reported good health, it was felt that the patient's a priori $25 \%$ risk, long standing IDDM, and recent development of exocrine insufficiency and weight loss placed her at high risk for developing pancreatic carcinoma. A prophylactic total pancreatectomy was performed.

The pancreas was densely fibrotic with extensive, heavily calcified, inspissated secretions throughout. Microscopically, there was widespread fibrosis of the pancreatic lobules accompanied by cystic dilatation of the lobular ducts, acinar atrophy, and periductal inflammation. Despite extensive sampling to identify neoplastic epithelium, there were only small foci of squamous metaplasia and mild atypia of ductal epithelium but no definite epithelial hyperplasia or dysplasia (fig 3 ).

The patient was stable during the initial postoperative period, but developed respiratory distress owing to non-cardiogenic pulmonary oedema on the second postoperative day, and required ventilatory assistance. In spite of aggressive efforts at support, her pulmonary oedema and respiratory distress remained intractable. At her family's request ventilatory assistance was discontinued and she died approximately five weeks postoperatively.

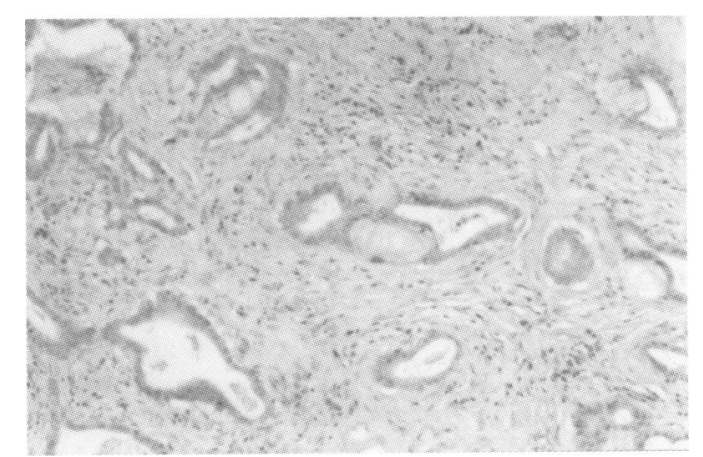

Figure 3 Case 2 (IV 19). Tissue submitted for K-ras proto-oncogene analysis. Pancreatic atrophy without hyperplasia or dysplasia is present within this lobule. The blue laminated structures in the lumina and walls of the ducts were calcified before the tissue was prepared for histology (HEE). 
K-ras mutations (codon 13 )

\begin{tabular}{llllll}
\hline Case & Tissue & \multicolumn{2}{l}{ Amino acid substitution } & Histology \\
\hline Case 1 (III-6) & Pancreas & $\begin{array}{l}\text { GGC } \\
\text { gly }\end{array}$ & $\rightarrow$ & $\begin{array}{l}\text { GAC } \\
\text { asp }\end{array}$ & Adenocarcinoma \\
Case 2 (IV-19) & Pancreas & $\begin{array}{l}\text { GGC } \\
\text { gly }\end{array}$ & $\rightarrow$ & $\begin{array}{l}\text { GAC } \\
\text { asp }\end{array}$ & $\begin{array}{l}\text { Squamous and mucinous } \\
\text { metaplasia }\end{array}$ \\
Case 3 (III-17) & Pancreas & $\begin{array}{l}\text { GGC } \\
\text { gly }\end{array}$ & $\rightarrow$ & $\begin{array}{l}\text { AGC } \\
\text { ser }\end{array}$ & Adenocarcinoma \\
Case 3 (III-17) & $\begin{array}{l}\text { Pancreatic } \\
\text { secretions }\end{array}$ & $\begin{array}{l}\text { GGC } \\
\text { gly }\end{array}$ & $\rightarrow$ & $\begin{array}{l}\text { AGC } \\
\text { ser }\end{array}$ & No cytological atypia \\
\hline
\end{tabular}

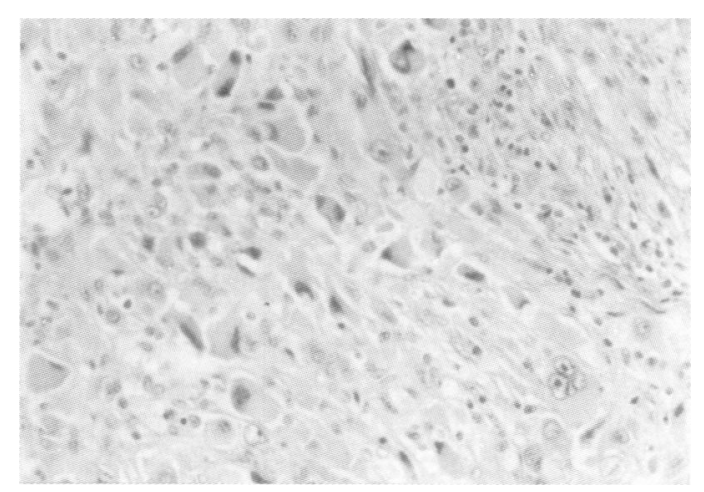

Figure 4 Case $3(I I I \cdot 17)$. Large cell anaplastic carcinoma. The pancreas also contained foci of small cell adenocarcinoma and moderately differentiated infiltrating ductal adenocarcinoma (HE्EE).

CASE 3 (III·17)

A 40 year old male cousin of the proband suffered from occasional abdominal pain for many years, usually temporally related to excessive ethanol consumption. One week before evaluation, he was found comatose and was diagnosed with diabetic ketoacidosis. A history compatible with pancreatic exocrine insufficiency was obtained.

Owing to his $50 \%$ a priori risk of pancreatic cancer and new diagnosis of IDDM, he was evaluated further. Abdominal CT scan showed a dense pancreas with no obvious masses. ERCP showed abnormal tortuous ectatic ducts, but was also non-diagnostic for cancer. $\mathrm{Cy}$ tological analysis of aspirated pancreatic fluid failed to show malignant cells. However, PCR analysis of the fluid indicated the presence of a mutated K-ras proto-oncogene (see Molecular analysis below). The patient elected to undergo a prophylactic pancreatectomy owing to his perceived high risk for developing pancreatic carcinoma.

His pancreas contained three geographically and histologically distinct invasive adenocarcinomas (large cell anaplastic, moderately differentiated infiltrating ductal, and poorly differentiated small cell types) in the midst of diffuse epithelial hyperplasia and dysplasia (fig 4). Carcinoma extended to one surgical margin and had metastasised to four lymph nodes. The same underlying pattern of acinar atrophy and fibrosis was present as was seen in the other two resected pancreata.

The patient survived his surgery and received outpatient chemotherapy for metastatic disease. with a minority of

analyses were repeated for

confirmation.
Molecular analysis

Exon 1 of the K-ras proto-oncogene was amplified and sequenced from DNA derived from malignant pancreatic tissue obtained from III 6 (case 1, fig 5) and III.17 (case 3), and from the non-malignant tissue derived from IV 19 (case 2). In addition to analysis of pancreatic tissue obtained at surgery, DNA extracted from pancreatic secretions obtained preoperatively by ERCP from III-17 (case 3) was analysed for the presence of K-ras mutations.

Mutations were found in pancreatic tissue from all three cases analysed, but not in constitutional tissue (lymphocytes and normal pancreatic tissue) (table). In patient III-17 the same mutation was found in ERCP fluid as was present in malignant pancreatic tissue obtained subsequently by surgical resection, thus confirming the validity of the preoperative diagnostic information in this patient. Moreover, it should be noted that histological analysis of pancreatic tissue of IV 19 showed no evidence of dysplasia or malignancy (fig 3), yet molecular analysis showed a mutation in the $\mathrm{K}$-ras protooncogene.

Surprisingly, the mutations in all cases were located in codon 13 (table). III. 6 and IV 19 had the same mutation at the second nucleotide (GGC to GAC) resulting in an amino acid change of glycine to aspartic acid, and III-17 had a mutation at the first nucleotide (GGC to AGC) resulting in an amino acid change of glycine to serine. No mutations were seen in codon 12 , the site at which the majority of human pancreatic cancers show K-ras point mutations.

\section{Discussion}

To our knowledge, the family described here represents the most extensive published report to date of a familial clustering of pancreatic carcinoma. In this pedigree, the propensity for developing pancreatic carcinoma appears to be inherited as a monogenic autosomal dominant trait with high penetrance. This family, coupled with previously published reports, ${ }^{4-11}$ clearly indicate that genetic factors can have a striking effect on the development of pancreatic malignancy.

In keeping with the pattern typically seen in families with a genetic predisposition to cancer, the mean age at diagnosis in members of this family (42 years) is substantially younger than the typical age at which people in the general population are diagnosed, where $80 \%$ of cases occur in people between the ages of 60 and $80 .^{2}$ The young age of onset provides strong evidence for a genetic predisposition in this family and is unique among reports of familial pancreatic cancer, the other reports having a relatively late age of onset in affected subjects. In addition to the striking pedigree structure and young ages at diagnosis, further compelling evidence of a genetic predisposition to pancreatic cancer in this family is provided by the multifocal nature of the cancer observed in case 3.

A strong association between IDDM and subsequent development of pancreatic cancer 
exists in this family. All nine people who developed pancreatic cancer had previously been diagnosed with IDDM; in five of these cases the diagnosis of diabetes preceded the diagnosis of malignancy by at least 10 years. Two of the three subjects who were diagnosed with IDDM (III. 3 and IV $\cdot 19$, case 2 ) died at ages less than 30 years old and thus would not have been expected to manifest cancer yet. The remaining person is currently 32 years old and again would probably be too young to manifest cancer.

The unique association of IDDM and adenocarcinoma of the pancreas in this family serves as a marker for the subsequent development of pancreatic carcinoma and may prove to be of considerable use in following members of this family, assessing their risks for malignant transformation, and guiding the timing and types of intervention to be used. As the proband and his cousin (case 3 ) illustrate, prophylactic surgery has a role in situations where genetic and clinical information indicate a high risk for development of a life threatening disease. The proband's initial course was encouraging, especially in the light of the fact that the surgical margins were free of disease. The recurrence of his disease 18 months postoperatively may have been secondary to rupture of a pancreatic cyst during surgery, with resultant spread of disease throughout the peritoneal cavity. The unfortunate case 2 illustrates the care that must be taken in considering prophylactic surgery as a means of prevention.

The underlying basis of the association in this family between IDDM and pancreatic cancer is unknown. Although an association between these two entities has been postulated to exist in the general population, ${ }^{12}{ }^{13}$ the relative risk of approximately 4-fold is modest and controversy exists regarding whether even this modest association is real. ${ }^{14}$ It thus seems unlikely that IDDM or pancreatic exocrine insufficiency has a causal relationship with pancreatic adenocarcinoma in this family, especially when one considers that the diagnosis of IDDM was made essentially concurrently with pancreatic carcinoma in four patients from this kindred. There is at present no known mechanism to explain how IDDM could result in pancreatic cancer although it has been shown that the diabetic state enhances growth of pancreatic carcinoma in vitro. ${ }^{15}$

The pathological features of the resected pancreata in this family suggest that IDDM, exocrine insufficiency, and neoplastic transformation are secondary consequences of an underlying abnormality. All three glands share the same distinctive and unusual pattern of widespread pancreatic destruction characterised by microcystic dilatation of intralobular ducts, progressive fibrotic replacement of the lobules, variable numbers of chronic inflammatory cells, and atrophy of acini. Islets and exocrine pancreatic tissue are both lost as a result of this process. Microscopic evidence of neoplastic progression is not present in every case and the histological appearance of hyperplastic/dysplastic epithelium differs between the two cases where it is present. The invasive carcinomas also differ strikingly in appearance between cases and within one case (case 3). The multiple carcinomas in case 3 appear to have originated independently and the presumed precursor lesions (hyperplasia, metaplasia, and dysplasia) are so widespread within the glands in the two cases with carcinoma as to suggest multifocal origin. These observations are consistent with the hypothesis that there is a generalised destructive process that predisposes to pancreatic cancer in this family, perhaps by stimulating reactive epithelial proliferation that serves to incorporate and perpetuate mutations that ultimately lead to carcinoma. There are reports that clinically evident pancreatitis increases the subsequent risk for pancreatic carcinoma, ${ }^{16}$ but no reports that clinically silent familial pancreatic destruction, such as we have observed, is a risk factor.

The pedigree reported here represents a unique opportunity to examine very early stages of pancreatic carcinogenesis in the human. The germline mutation that is responsible for the predisposition to pancreatic cancer in this family is unknown. However, since the K-ras protooncogene is mutated in 75 to $90 \%$ of cases of sporadic human pancreatic cancer, ${ }^{1718}$ it seemed potentially instructive to analyse the integrity of this gene in the pancreatic tumours from people within this family with the thought that mutations in ras might contribute to neoplastic progression. In pancreatic tissue from all three people analysed, K-ras point mutations were present, serving to illustrate that fundamental similarities exist between the pancreatic cancer observed in this family and that observed in the general population.

Several issues regarding the molecular changes observed in this family are worthy of note. All three mutations identified in the pancreatic tissue from these family members were localised to codon 13 . This is a striking observation since only a minority (less than $10 \%$ ) of human pancreatic cancer associated $\mathrm{K}$ ras mutations occur in codon 13 , the majority being found in codon 12 . This suggests that in the context of these people's inherited predisposition to pancreatic cancer, there may be a selective advantage to cells which have specifically mutated codon 13 . The mechanism of this phenomenon may be related to a specific interaction between K-ras and the (as yet unidentified) gene product which is mutated in the germline of this family. The observation of several different $\mathrm{K}$-ras mutations in pancreatic tumours from this family, and the presence of only wild type $\mathrm{K}$-ras in normal tissue, clearly show that a mutant K-ras gene does not represent the primary (germline) event which is responsible for the pancreatic cancer in this family. However, K-ras mutations seem to occur early in the pathogenesis of their pancreatic cancer, given that a mutated $\mathrm{K}$-ras allele was present in pancreatic tissue from case 2 which did not yet show histological evidence of malignancy. Together with her history of diabetes, the presence of a K-ras mutation in her pancreatic tissue provides compelling evidence that case 2 inherited the pre- 
disposition to cancer; thus the pathological findings in her serve to illustrate the early stages of pancreatic transformation which precede the onset of overt malignancy in this family. The observation of a K-ras mutation in non-dysplastic pancreatic tissue has not been previously reported, and supports the hypothesis that mutations in K-ras are a very early event in human pancreatic cancer.

Even in subjects at high risk for pancreatic cancer, detection of malignancy is difficult during the early stages at which surgery might be curative. We and others ${ }^{19-21}$ have detected $\mathrm{K}$ ras mutations in pancreatic duct aspirates obtained at ERCP in patients with pancreatic adenocarcinoma. In the present report, this has been achieved in a prospective manner in a person at risk. Such screening may provide a powerful means of identifying people in this family who have inherited the predisposition to cancer and have begun to accrue the genetic changes which ultimately lead to malignancy. The presence of a K-ras mutation in case 2 offers the possibility that such detection may be feasible at a very early stage, even before the development of histologically diagnostic neoplastic changes. Given the sensitivity of PCR, less invasive means than ERCP for obtaining pancreatic secretions (such as duodenal aspiration) may prove feasible. The question of what to do with information thus obtained, however, is more problematical. The postoperative death of case 2 serves to illustrate the gravity with which therapeutic options must be considered in such families.

In summary, we report an extensive kindred in which a propensity for pancreatic cancer is transmitted as an autosomal dominant trait. IDDM and exocrine insufficiency serve as early phenotypic markers to indicate who has inherited the aberrant allele. Molecular detection of K-ras mutations in cells obtained by ERCP allowed the preclinical identification of a person who had inherited the predisposition to pancreatic cancer. Further study of this family may shed light on the aetiology of pancreatic carcinoma in this family, its pathogenesis in the general population, and the relationship between IDDM and pancreatic cancer.

JPE is supported through the Lucille P Markey Molecular Medicine Center at The University of Washington. We would like to thank Mary Bronner, Rodger Haggitt, and Sharon Plon for helpful suggestions and comments.

1 Ponder BAJ. Inherited predisposition to cancer. Trends Genet 1990;6:213-18.

2 Boring CC, Squires TS, Tong T. Cancer statistics. $C A$ Cancer f Clin 1993;43:7-26.

3 Goelz SE, Hamilton SR, Vogelstein B. Purification of DNA from formaldehyde fixed and paraffin embedded human tissue. Biochem Biophys Res Commun 1985;130:118-25.

4 MacDermott RP, Kramer P. Adenocarcinoma of the pancreas in four siblings. Gastroenterology 1973;65:137-9.

5 Dat NM, Sontag SJ. Pancreatic carcinoma in brothers. Ann Intern Med 1982;97:28

6 Ehrenthal D, Haeger L, Griffin T, Compton C. Familial pancreatic adenocarcinoma in three generations. Cancer 1987;59:1661-4

7 Lynch H, Fitzsimmons ML, Smyrk TC, et al. Familial pancreatic cancer: clinical pathologic study of 18 nuclear families. Am $\mathcal{F}$ Gastroenterol 1990;85:54-60.

8 Danes BS, Lynch HT. A familial aggregation of pancreatic cancer: an in vitro study. $\mathcal{F} A m$ Med Assoc 1982;247. 2798-802.

9 Friedman JM, Fialkow PJ. Familial carcinoma of the pancreas. Clin Genet 1976;9:463-9.

10 Reimer RR, Fraumeni JF Jr, Ozols RF, Bender R. Pancreatic cancer in a father and son. Lancet 1977;i:911-12.

11 Appel MF. Hereditary pancreatitis. Arch Surg 1974;108. 63-5.

12 Cuzik J, Babiker AG. Pancreatic cancer, alcohol, diabetes mellitus, and gall-bladder disease. Int f Cancer 1989;43: 415-21.

13 Adami HO, McLaughlin J, Ekbom A, et al. Cancer risk in patients with diabetes mellitus. Cancer Causes Control 1991; 2:307-14.

14 Gullo L, Pezzilli R, Morselli-Labate AM. Italian Pancreatic Cancer Study Group. Diabetes and the risk of pancreatic cancer. N Engl 7 Med 1994;331:81-4

15 Fisher W, McCullough P, Ramo O, Bell R. Further studies of enhanced growth of pancreatic carcinoma in diabetes. Surg Res 1990;48:403-7.

16 Lowenfels AB, Maisonneuve P, Cavallini G, et al. Pancreatitis and the risk of pancreatic cancer. $N$ Engl $f \mathrm{Med}$ 1993;328:1433-7

17 Almoguera C, Shibata D, Forrester K, Martin J, Arnheim N, Perucho M. Most human carcinomas of the exocrine pancreas contain mutant c-K-ras genes. Cell 1988;53: $549-54$

18 Motojima K, Urano T, Nagata Y, Shiku H, Tsunoda T, Kanematsu T. Mutations in the Kirsten-ras oncogene are common but lack correlation with prognosis and tumor stage in human pancreatic carcinoma. Am $\mathcal{f}$ Gastroenterol 1991;214:652-7.

19 Brentnall T, Crispin D, Byrd D, et al. K-ras mutations detected in pancreatic fluid not diagnosed by conventional techniques. Gastroenterology 1993;104.

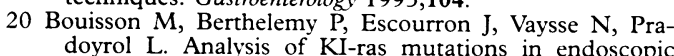
retrograde cholangio-pancreatography (ERCP) samples. Gastroenterology 1993;104.

21 Tadu M, Omata M, Kawai S, et al. Detection of ras gene mutations in pancreatic juice and peripheral blood of patients with pancreatic adeno-carcinoma. Cancer Res 1993;53:2472-4. 